\title{
Overturning and rolling avoidance by gecko when challenged with inclines
}

\author{
Qiang Xing ${ }^{\mathrm{a}, *}$, Zhouyi Wang ${ }^{\mathrm{b}}$, Weijia Zong ${ }^{\mathrm{b}}$ and Jun Gu ${ }^{\mathrm{a}}$ \\ ${ }^{a}$ School of Mechanical Engineering, Nantong University, 9 Seyuan Road, Nantong, \\ Jiangsu 226019, P.R. China \\ ${ }^{\mathrm{b}}$ Institute of Bio-inspired Structure and Surface Engineering, Nanjing University of \\ Aeronautics and Astronautics, 29 Yudao Street, Nanjing, Jiangsu 210016, P.R. China \\ *Corresponding author's e-mail address: meexq@ntu.edu.cn
}

Received 6 September 2019; initial decision 17 December 2019; revised 18 March 2020; accepted 16 April 2020; published online 6 May 2020

\begin{abstract}
Understanding how animals avoid overturning and rolling in motion to maintain movement stability and to accommodate their habitat and the mechanisms of movement in these habitats is a matter of concern. Gecko climbs a more inclined substrate by lowering the speed; meanwhile, the duty factor is increased with the increase of the incline angle, indicating that the gecko switches the diagonal gait when climbing on the shallow inclines to the triangular gait when on the inverted surface. The overturning impulse moment is increased with an increase of the incline angle. On inclines larger than $90^{\circ}$, the positive and negative overturning impulse moments are increased significantly and show obvious differences. The maximum value of rolling impulse moment on the surface at $180^{\circ}$ can reach 15 times that of the minimum value on the surface at $90^{\circ}$, and the positive and negative rolling impulse moments at the inclined surface of $120-180^{\circ}$ have obvious differences. The above results show that on shallow inclines, the low centre of mass and the flat posture of the gecko can effectively improve locomotion stability; simultaneously, through the timely conversion of the limb function, the overturning/rolling impulse moments are low, which greatly reduces the probability of overturning/rolling during locomotion. However, on inverted inclines, the gecko takes full advantage of the flexibility of body and limbs to delay the occurrence of rolling and overturning, and actively cooperates with the adjustment of the gait, using the alternating change of gait to avoid overturning and rolling.
\end{abstract}

\section{Keywords}

overturning, rolling, incline, gecko, avoidance strategy. 


\section{Introduction}

Animal motion patterns (locomotion modes) (flight, swimming, running) are determined by the intrinsic motion function systems (neural, musculoskeletal, etc.) of the entire motor system and their interaction with the environment (Wakeling \& Johnston, 1999; Dickinson et al., 2000). Locomotor behaviour is an external characterization of the interaction of the motion system and its intrinsic properties. Different environments place different requirements on animal movement systems (Bi et al., 2015; Taylor, 2018). The natural habitat of most terrestrial legged animals is filled with mixed substrates, including sloping terrain, rocks, dead or prosperous plants; therefore, animals need to move or circumvent on inclined surfaces frequently, especially small ones. In order to completely understand how small animals adapt to their habitat and their movement mechanisms for these habitats, it is necessary to study the biomechanics and behaviour of animals on various inclined surfaces; besides, this provides reference and inspiration for the mechanism design, gait planning and control system design of bionic robot, as well as support for revealing the evolutionary laws of animal motion function.

Faced with the challenge of inclined locomotion, the distance between the centre of mass (COM) and the substrate increases with the incline angle, and then the required force between the foot and the substrate are increased, resulting in the a higher possibility of overturning or lateral rolling (Herr et al., 2002; Lammers \& Gauntner, 2008; Lee, 2011; Bi et al., 2015). Much attention has been drawn to the issue of how animals avoid overturning and rolling during locomotion to keep their movements stable when encountering external disturbances (climbing or overstepping obstacles). By increasing the distance between its stance phases, the horizontally walking octopus crab balances the overturning and rolling moments caused by fluid interference (Martinez et al., 1998). Hexapod insects moving in horizontal or inclined surface can avoid overturning by the real-time adjustment of the reaction force (RF) of each walking leg, so as to achieve the passive dynamic balance in the movement (Ting et al., 1994; Goldman et al., 2009); and the overturning moment can also be reduced by the backward movement of the COM (Holmes et al., 2006). Erect quadruped animals use crouching movement during exercise and adjust the normal RF of the front and hind legs to reduce or balance the overturning moment (Lee et al., 1999; Herr et al., 2002; Lammers et al., 2006). The gecko (Gekko gecko) increases the stability of the movement by increasing the fore-aft distance of the supporting limbs in 
the vertical-plane movement, thereby improving the motion stability; during the actual locomotion, the gecko can keep the motion stability through the real-time adjustment in RF of the limbs (Autumn et al., 2006; Wang et al., 2011). Meanwhile, the tail also plays an important role in avoiding overturning on vertical substrate (Jusufi et al., 2008). Previous studies have found that tree lizards (Anolis carolinensis) actively change their posture to adapt to changes in environmental inclination, but to better overcome an instability (overturning moment) caused by the change of the inclines and provide more driving force, the pattern of limb movement reaction is also being changed correspondingly (Krause \& Fischer, 2013).

As a species of crawling animal with excellent climbing ability, the research on gecko's athletic ability helps to understand the mechanism of adhesion movement and the evolution of climbing motion function. Many studies have been devoted to the study of the characteristics of the bristle structure, the adhesion mechanism, and the kinematics and dynamics of the inclined climbing to explore its excellent adhesion climbing ability; these studies have also inspired the design of the bionic wall-climbing robot. However, there are still problems that are not perfectly explained, and the internal mechanism needs further exploration, such as: (1) Investigating the effects of the change of inclination, especially on the steep inclines (Incline angle $>60^{\circ}$ ), in the gecko's coordination of behaviour and RF of the limbs, to avoid overturning. (2) The high stability of the tripod gait of the hexapod insect can avoid the lateral rolling in the locomotion; however, geckos are four-limbed, and the influence of diagonal gait adoption on the rolling movement in climbing must be explored, especially in the case of the RF rapidly increasing on the steep inclines. To solve these issues, the locomotor behaviour and mechanics of geckos on different inclines were investigated in this paper, and then some interesting issues were discussed, such as: how the gecko can prevent overturning and rolling in the movement through the coordinated control of force and behaviour in the climbing locomotion, and whether the regulatory strategy will have a transition threshold due to the difference in incline angles.

\section{Materials and methods}

\subsection{Experimental materials}

\subsubsection{Animals}

Eight Gekko geckos (reptilian, lizard, gecko) produced in Guangxi province (P.R. China) (body weight: $63.4 \pm 2.6 \mathrm{~g}$; the snout-to-vent length: $132.4 \pm$ 
$10.3 \mathrm{~mm}$; mean $\pm \mathrm{SD}$ ) were used in the experiment. They were raised in a room with a supply of bread bug, cockroaches, vitamin materials and water, and the room was under simulated natural conditions including the natural light, air conditioning $\left(25 \pm 2^{\circ} \mathrm{C}\right)$ and a humidity regulator $(60-70 \%)$.

\subsubsection{Experimental setup}

An animal locomotor behaviour-reaction force measuring system consists of two core modules: a force measuring module and a behaviour recording module (Figure 1A) (Dai et al., 2011). The force measuring module includes a force sensor array $($ FMA) consisting of $24(3 \times 8) 3 \mathrm{D}$ force sensors with a resolution of $\mu \mathrm{N}$, the NI PXI series signal conditioning system, a set of

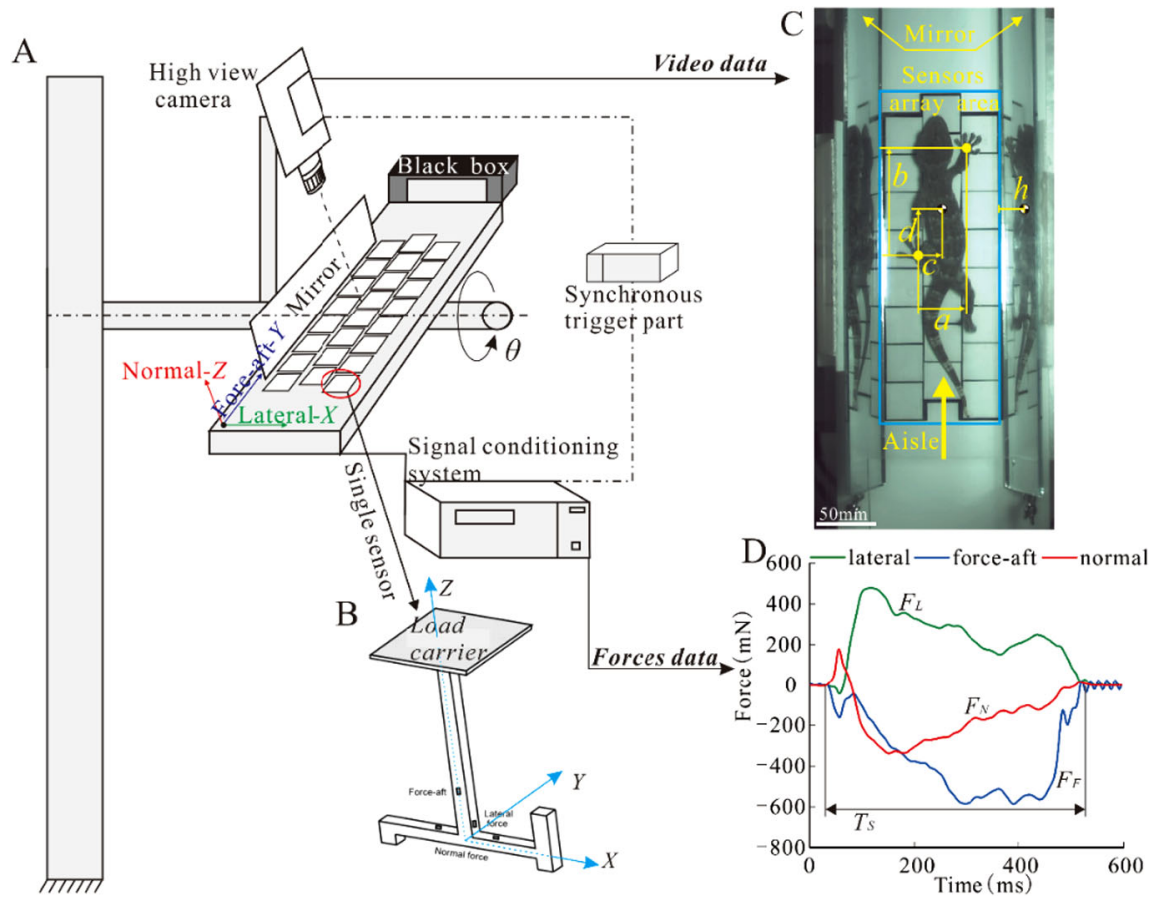

Figure 1. Experimental equipment and procedure. (A) Animal locomotive behaviour and reaction force measurement system; the aisle of the reaction force measurement was rotated (each step $30^{\circ}$ ) in order to simulate a gecko moving over different inclined substrates ( $\theta$ : inclined angle of aisle substrate). (B) Structural diagram of a single 3-dimensional sensor. (C) When a gecko moves through an aisle of the FMA, the vertical view and two side-views in mirrors of locomotive behaviours were recorded by a video camera located perpendicular to the FMA at 250 frames per second. (D) Three-dimensional reaction force (RF) versus time during a single stance phase of a Gekko gecko moving on a substrate sloping at a $180^{\circ}$ angle. 
experimental test software based on the LabVIEW platform, etc. A carrier sheet $(30 \times 30 \mathrm{~mm})$ was attached to the top end of the force sensor (Figure 1B) which formed the motion surface of the animal. In order to avoid the mutual influence of the adjacent sensors of the force measuring array in the actual test, the deformation of each sensor under the maximum load was estimated, and the carrier sheets at the ends of the adjacent sensors were separated by $1.0 \pm 0.1 \mathrm{~mm}$. The animal motion reaction force signal is filtered using a Butterworth low-pass filter with a cut-off frequency of $100 \mathrm{~Hz}$. In order to achieve synchronous acquisition of image data and mechanical data, a synchronous trigger module is used to trigger high-speed cameras (ispeed-3, Olympus, Tokyo, Japan) and force measuring system. To measure the motion reaction of the gecko in response to changes of the inclined planes, the entire force channel can be rotated about a horizontal axis on the frame, simulating different inclines of any inclination from $0^{\circ}$ (horizontal plane-ground) to $180^{\circ}$ (inverted plane-ceiling). The high-speed camera is always perpendicular to the FMA channel plane and the viewing range covers the entire array. A pair of plane mirrors on the left and right sides of the sensor array makes it possible to obtain the animal's three-dimensional locomotor behaviour (150 500 fps, Figure 1C) through only one high-speed camera - the real images of the lateral and fore-aft direction locomotor behaviour as well as the virtual side-view images of the normal and fore-aft direction. A black box was placed at the end of the channel to induce the geckos (Figure 1A).

\subsection{Data processing}

To obtain the relative geometrical position on different ramps, the gecko's locomotor behaviour was recorded with a high-speed camera (250 fps) (Figure 1A). The motion reaction-force data and the image data recording the motion behaviour were checked to make sure that there was no sudden pause in the selection test, the whole experimental process was smooth, and there was no slip between the sole and the substrate. The position of the COM of gecko was located through the reaction board method (Lammers et al., 2006). Prior to the experiment, the COM markers were painted on the back of the gecko. The coordinates of the reference point in the gecko movement were obtained from the experimental video using the i-SPEED Viewer software ( $i$-SPEED 3, Olympus), and the corresponding motion parameters were calculated: the forward speed $v$ (only the trials when the velocity close to 
uniform speed and the change of velocity no more than $15 \%$ of its average in trial was selected and further evaluated); The duty factor $D_{\mathrm{f}}$ (=ratio of the stance phase to a gait cycle); lateral distance of the supporting foot $\vec{a}$ (the lateral coordinate difference of the stance phase in each step, from the hind support phase to the front support phase in the lateral direction); foreaft distance of supporting foot $\vec{b}$ (the fore-aft coordinate difference of the support phase in each step, from the hind support phase to the front support phase in the fore-aft direction); the distance between the COM and the surface $\vec{h}$ (the normal coordinate of the COM in motion, from the substrate to the COM); During the movement, the lateral displacement of the COM $\vec{c}$ (the lateral coordinate of the COM and the lateral coordinate of the supporting hind leg, it and $\vec{a}$ in the same direction); the fore-aft displacement of the COM $\vec{d}$ (the fore-aft coordinate of the COM and the fore-aft coordinate of the supporting hind leg, it and $\vec{b}$ in the same direction) (Figure 1C). Because the position of the artificially acquired feature points unavoidably have errors during the processing, the obtained parameter data is not continuous enough, so it must be processed by means of the filtering tool. This paper used the digital smoothing filter tool (Biotest, Germany) to remove the data jitter. The characteristics of the original curve are preserved, and the filtered data is statistically analysed.

\subsubsection{Overturning and rolling impulse moments}

When gecko moves on the substrate, the force acting on each foot of gecko produces a moment, which causes the body to roll or overturn. However, the occurrence of overturning or rolling is ultimately determined by whether the external load input is in a state of motion instability, which is a time accumulation effect of external load. Different from the instantaneous force/moment, impulse moment is a physical quantity that describes the cumulative effect of force acting on the particle for a period of time. Its magnitude is equal to the product of the external moment acting on the object and the acting time (the direction is the same as the moment) or the product of the impulse acting on the object and the force arm (the direction is the same as the impulse). It can be used to describe the changes in the rotating state of the object and provide an additional bio-mechanical process information for the study of stability control mechanism.

Figure 1D shows the relationship between the reaction force (RF) acting on the left front foot in a support phase $\left(T_{\mathrm{S}}\right)$ and the time when the gecko is free to climb on the vertical plane: the negative force in the fore-aft direction 
$\left(F_{\mathrm{F}}\right)$ indicates that the limb hinders the movement; The positive lateral force $\left(F_{\mathrm{L}}\right)$ on the right front foot indicates that the right hind limb pulls the body to the right; when the normal force $\left(F_{\mathrm{N}}\right)$ is negative, it indicates that the sole produces adhesion to pull the body toward the substrate (Figure 1D). The animal's locomotor behaviour is the result of the continuous action of the $\mathrm{RF}$ on the foot. In order to demonstrate the contribution of the walking leg to avoid the overturning and rolling movements, the impulses of the feet's RF in the three directions before and after the whole supporting phase is calculated as following (Eq. 1):

$$
I_{\mathrm{L}}=\int_{0}^{T_{\mathrm{S}}} F_{\mathrm{L}} \mathrm{dt}, \quad I_{\mathrm{F}}=\int_{0}^{T_{\mathrm{S}}} F_{\mathrm{F}} \mathrm{dt}, \quad I_{\mathrm{N}}=\int_{0}^{T_{\mathrm{S}}} F_{\mathrm{N}} \mathrm{dt}
$$

During the locomotion, the centre of mass of the gecko always has a distance $h$ from the motion plane, and as the inclination of the planes increases, the overturning due to the deviation of the COM from the substrate will occur (Figure 2A). The supporting phase is constantly reciprocating, and the motion RF is constantly changing, from nonexistence to existence, from existence to nonexistence, which results in the imbalance of the RF of the left

A

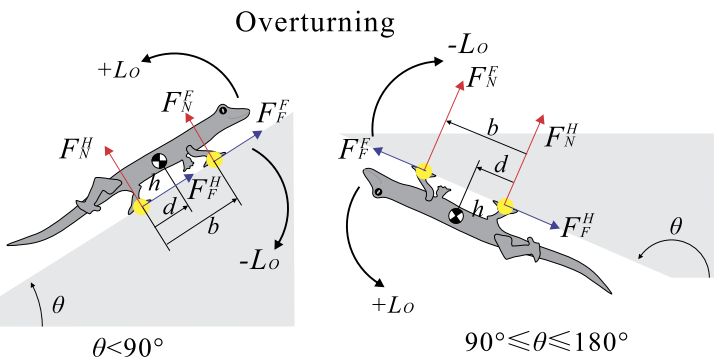

B

Rolling

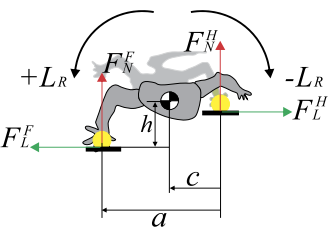

$\theta<90^{\circ}$

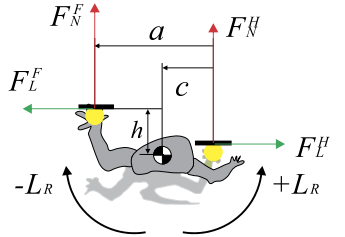

$90^{\circ} \leqslant \theta \leqslant 180^{\circ}$

Figure 2. Schematic diagrams of calculation of gecko overturning impulse moment and rolling impulse moment on inclines (A) overturning impulse moment on inclines; (B) rolling impulse moment on inclines. 
and right feet and will produce a lateral roll around the fore-aft axis of the body (Figure 2B). At the same time, the gecko also generates a corresponding stabilizing moment (impulse moment) by adjusting the motion posture and the RF of the walking foot, etc., to avoid overturning and rolling, thereby ensuring the stability of the movement. In accordance with what happens in nature (the true movement of the gecko on different inclines), the axis of rotation is taken through the COM. To reveal the balance strategy for overcoming overturning and rolling in gecko movement to maintain stability, the positive and negative overturning impulse moments $\left(+L_{\mathrm{O}}\right.$ and $\left.-L_{\mathrm{O}}\right)$ and positive and negative rolling impulse moments $\left(+L_{R}\right.$ and $\left.-L_{R}\right)$ in the onestep motion of the gecko are calculated by equations (2)-(5).

$$
\begin{gathered}
+L_{\mathrm{O}}=\left\{\begin{array}{cc}
\mid \int_{0}^{T_{\mathrm{S}}} \vec{h} \times \vec{F}_{\mathrm{F}}^{\mathrm{F}} \mathrm{dt}+\int_{0}^{T_{\mathrm{S}}} \vec{h} \times \vec{F}_{\mathrm{F}}^{\mathrm{H}} \mathrm{dt} \\
+\int_{0}^{T_{\mathrm{S}}}(\vec{b}-\vec{d}) \times \vec{F}_{\mathrm{N}}^{\mathrm{F}} \mathrm{dt} \mid & \theta \in\left[0^{\circ}, 60^{\circ}\right] \\
\left|\int_{0}^{T_{\mathrm{S}}} \vec{h} \times \vec{F}_{\mathrm{F}}^{\mathrm{F}} \mathrm{dt}+\int_{0}^{T_{\mathrm{S}}} \vec{d} \times \vec{F}_{\mathrm{N}}^{\mathrm{H}} \mathrm{dt}\right| & \theta \in\left[90^{\circ}, 180^{\circ}\right]
\end{array}\right. \\
-L_{\mathrm{O}}= \begin{cases}\left|\int_{0}^{T_{\mathrm{S}}} \vec{d} \times \vec{F}_{\mathrm{N}}^{\mathrm{H}} \mathrm{dt}\right| & \theta \in\left[0^{\circ}, 60^{\circ}\right] \\
\left|\int_{0}^{T_{\mathrm{S}}} \vec{h} \times \vec{F}_{\mathrm{F}}^{\mathrm{H}} \mathrm{dt}+\int_{0}^{T_{\mathrm{S}}}(\vec{b}-\vec{d}) \times \vec{F}_{\mathrm{N}}^{\mathrm{F}} \mathrm{dt}\right| & \theta \in\left[90^{\circ}, 180^{\circ}\right]\end{cases} \\
+L_{\mathrm{R}}= \begin{cases}\left|\int_{0}^{T_{\mathrm{S}}}-\vec{c} \times \vec{F}_{\mathrm{N}}^{\mathrm{H}} \mathrm{dt}+\int_{0}^{T_{\mathrm{S}}} \vec{h} \times \vec{F}_{\mathrm{L}}^{\mathrm{H}} \mathrm{dt}\right| & \theta \in\left[0^{\circ}, 60^{\circ}\right] \\
\left|\int_{0}^{T_{\mathrm{S}}}-\vec{c} \times \vec{F}_{\mathrm{N}}^{\mathrm{H}} \mathrm{dt}+\int_{0}^{T_{\mathrm{S}}} \vec{h} \times \vec{F}_{\mathrm{L}}^{\mathrm{F}} \mathrm{dt}\right| & \theta \in\left[90^{\circ}, 180^{\circ}\right]\end{cases} \\
-L_{\mathrm{R}}= \begin{cases}\left|\int_{0}^{T_{\mathrm{S}}}(\vec{a}-\vec{c}) \times \vec{F}_{\mathrm{N}}^{\mathrm{F}} \mathrm{dt}+\int_{0}^{T_{\mathrm{S}}} \vec{h} \times \vec{F}_{\mathrm{L}}^{\mathrm{F}} \mathrm{dt}\right| & \theta \in\left[0^{\circ}, 60^{\circ}\right] \\
\left|\int_{0}^{T_{\mathrm{S}}}(\vec{a}-\vec{c}) \times \vec{F}_{\mathrm{N}}^{\mathrm{F}} \mathrm{dt}+\int_{0}^{T_{\mathrm{S}}} \vec{h} \times \vec{F}_{\mathrm{L}}^{\mathrm{H}} \mathrm{dt}\right| & \theta \in\left[90^{\circ}, 180^{\circ}\right]\end{cases}
\end{gathered}
$$

In equations (2)-(5), $F_{\mathrm{L}}^{\mathrm{H}}, F_{\mathrm{F}}^{\mathrm{H}}$ and $F_{\mathrm{N}}^{\mathrm{H}}$ are the lateral force, the fore-aft force, and the normal force acting on the hind foot, respectively. Similarly, $F_{\mathrm{L}}^{\mathrm{F}}, F_{\mathrm{F}}^{\mathrm{F}}$ and $F_{\mathrm{N}}^{\mathrm{F}}$ are the lateral force, the fore-aft force, and the normal force acting on the front foot, respectively.

\subsection{Statistics}

We have selected data from more than 1000 trials. Only trials in which the gecko moved at a near-steady forward speed were evaluated further. All geometric data and mechanical data were normalized before the data was statistically analysed. Specifically, the length data was divided by the body length of the experimental animal, the unit was BL; the force data was divided by the weight value of the test animal, the unit was BW, and the impulse was converted into BWs, the moment of the moment was converted to BWBLs. All individual measured data were combined and analysed using statistical 
software SPSS (version 15.0, SPSS, Chicago, IL, USA). The experimental data of the motion planes with the inclination angle ranging from 0 to $180^{\circ}$ were compared, and the covariance (ANCOVA) was used to obtain the $p$ value, in which the inclination angle was set as an independent variable, and the independent variables included speed, duty ratio, lateral distance, foreaft distance, normal deviation, lateral force impulse, fore-aft force impulse, normal force impulse, overturning impulse moment and rolling impulse moment. For each covariance analysis performed, the covariates were set to the experimental animal group and the locomotion speed. Since the animals used for the seven inclinations were different, we did not perform a repeated measurement covariance analysis. When $p<0.05$, the difference was significant. The data tested were expressed as mean \pm standard deviation (mean \pm $\mathrm{SD})$.

\subsection{Abbreviations}

COM Centre of mass

RF Reaction force

$v$ Forward speed

$\theta$ Inclination angle of inclined substrate

$D_{\mathrm{f}}$ Duty factor

$\vec{a}$ The lateral coordinate difference of the stance phase in each step, from the hind support phase to the front support phase in the lateral direction

$\vec{b}$ The fore-aft coordinate difference of the support phase in each step, from the hind support phase to the front support phase in the fore-aft direction

$\vec{c}$ The lateral coordinate of the COM and the lateral coordinate of the supporting hind leg, it and $\vec{a}$ in the same direction

$\vec{d}$ The fore-aft coordinate of the COM and the fore-aft coordinate of the supporting hind leg, it and $\vec{b}$ in the same direction

$\vec{h}$ The normal coordinate of the COM in motion, from the substrate to the $\mathrm{COM}$

$F_{\mathrm{L}}$ Lateral reaction force

$F_{\mathrm{F}}$ Fore-aft reaction force 
$F_{\mathrm{N}}$ Normal reaction force

$I_{\mathrm{L}}$ The lateral impulses acting on foot during stance phase

$I_{\mathrm{F}}$ The fore-aft impulses acting on foot during stance phase

$I_{\mathrm{N}}$ The normal impulses acting on foot during stance phase

$+L_{\mathrm{O}}$ and $-L_{\mathrm{O}}$ The positive and negative overturning impulse moments

$+L_{\mathrm{R}}$ and $-L_{\mathrm{R}}$ The positive and negative rolling impulse moments

\subsection{Data availability and ethical statements}

The data used to support the findings of this study are available from the corresponding author upon request.

This study was carried out accordance with the Guide of Laboratory Animal Management Ordinance of China. The experimental procedures were approved by the Jiangsu Association for Laboratory Animal Science (Jiangsu, P.R. China).

\section{Results}

\subsection{Locomotor behaviours}

From the overall trend of speed change, the gecko climbed to a more inclined substrate by lowering speed $\left(v=-0.002 \theta+0.937, R^{2}=0.491\right.$, $\mathrm{df}=178$, $p<0.001$ ) (Figure 3A). When the inclined angle was changed from 0 to $60^{\circ}$ (shallow inclines), there was no significant decrease in speed, and the average speed was about $0.84 \pm 0.19 \mathrm{~m} / \mathrm{s}$. Similarly, there was no significant change in the speed of movement in inverted inclines (incline angle $\geqslant 150^{\circ}$ ), and the average speed was about $0.33 \pm 0.15 \mathrm{~m} / \mathrm{s}$. Two distinct speed attenuations occurred between 60 and $90^{\circ}$ and between 120 and $150^{\circ}$. Specifically, the velocity was $0.55-1.12 \mathrm{~m} / \mathrm{s}$ at $0^{\circ}$ surface, the step frequency was $7.4-10.0 \mathrm{~Hz}$, and the step was 141-198 mm. The effect of speed on the duty factor was not significant ( $p=0.183$ ): at $30^{\circ}$ surface, the speed was $0.60-1.25 \mathrm{~m} / \mathrm{s}$, the step frequency was $4.9-9.3 \mathrm{~Hz}$, the step was $148-205 \mathrm{~mm}$, and the speed had no significant effect on the duty factor $(p=0.182)$; the speed was $0.71-$ $1.46 \mathrm{~m} / \mathrm{s}$ at $60^{\circ}$ surface, the step frequency was $6.7-8.6 \mathrm{~Hz}$, and the step was $150-190 \mathrm{~mm}$. There was a significant exponential relationship between the duty factor and the speed $(p<0.001)$, which was decreased with increasing 

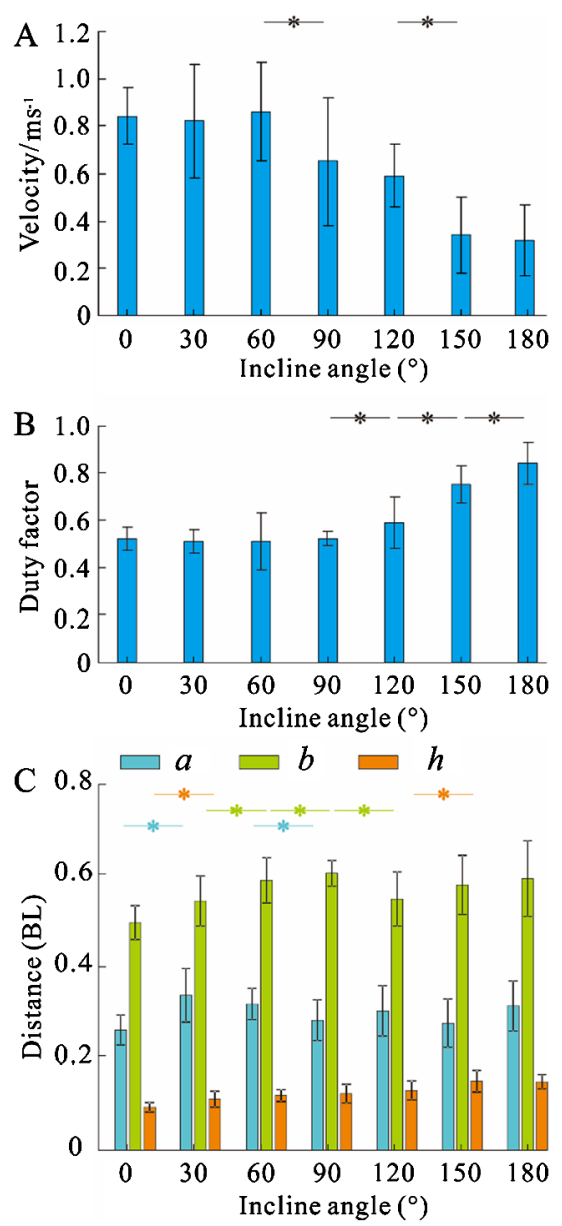

Figure 3. Statistical mean values of kinematic parameters of geckos on different inclines. (A) Velocity attenuates from $90^{\circ}$ incline angle, and there is no significant difference between 150 and $180^{\circ}$. (B) Duty factor. (C) Posture parameters. An asterisk indicates that there is a significant difference between the corresponding parameters in different inclines.

speed $($ duty factor $=1.3717 \times \exp (-0.8749 \times v))$; the velocity was $0.29-$ $0.77 \mathrm{~m} / \mathrm{s}$ at $90^{\circ}$ surface, the step was $84.6-144.9 \mathrm{~mm}$, and the step frequency was $0.73-6.00 \mathrm{~Hz}$, and there was no significant correlation between duty factor and speed $(p=0.158)$. The motion velocity at $120^{\circ}$ surface was $0.32-$ $0.86 \mathrm{~m} / \mathrm{s}$, the step frequency was $4.67-7.8 \mathrm{~Hz}$, and the variation range of the gecko crawling step was $93-158 \mathrm{~mm}$. There was a significant correlation between duty factor and speed $(p=0.022)$; this means that with an increasing 
speed the duty factor was decreased (duty factor $=-0.285 \times v+0.813$ ); the climbing speed was $0.14-0.66 \mathrm{~m} / \mathrm{s}$ at $150^{\circ}$ surface, the step frequency range was $1.9-5.1 \mathrm{~Hz}$, the variation range of the gecko crawling step was 79-135 mm, and there was a significant correlation between the duty factor and the velocity $(p=0.018)$, which increased with the increase of the speed (duty factor $=0.3172 \times v+0.638$ ). The tripod gait was dominant, the phase of the diagonal foot movement was considerably different, and the motion phase of the legs in the same side was not completely opposite. The speed range was $0.17-0.48 \mathrm{~m} / \mathrm{s}$, and the load coefficient had no significant correlation with the speed ( $p=0.302$ ) (Figure 3A, B) (Wang et al., 2014).

The change of the gecko's posture parameters played an important role in the unstable balance during the movement. The gecko constantly adjusted the space distance between the supporting feet $(a, b)$ to adapt to the change of the inclination angle. There was no significant linear regression between the incline angle and the lateral and fore-aft distances $(a, b)(p=0.836$ and $p=0.077)$. The overall trend was that as the inclined angle was increased, the gecko continued to extend its limbs to the outside to make the body more and more flat. The lateral distance of the supporting feet $(a)$ reached the minimum and maximum values at $90^{\circ}$ and $180^{\circ}$, respectively; from 0 to $30^{\circ}$, $a$ was significantly increased $(p<0.001)$, and from $60^{\circ}$ to $90^{\circ}, a$ showed a significant decrease trend $(p=0.031)$. The fore-aft distance between the supporting feet fluctuated with the incline angle. As the inclination was increased from 0 to $90^{\circ}$, the fore-aft distance $(b)$ between the limbs of the supporting feet increased remarkably; and with the inclination changed from 90 to $120^{\circ}, b$ obviously showed a significant decrease trend $(p=0.002)$, and the surface of $120-180^{\circ}$ showed an increasing trend. The overall trend of the distance from the substrate to the $\mathrm{COM}(h)$ was increased as the inclined angle was increased $\left(h=0.009 \theta+0.102, R^{2}=0.451\right.$, df $\left.=178, p<0.001\right)$, but on the inclined angle from 0 to $30^{\circ}$ and from 120 to $150^{\circ}$, there was a significant increasing trend ( $p=0.008$ and $p=0.001$ ) (Figure 3C).

\subsection{The impulse of the force of single foot}

In the stable locomotion on inclines of the gecko, the lateral impulse of the front and hind feet was increased with the increase of the inclination, and the increase of the lateral impulse of the front foot $\left(I_{\mathrm{L}}^{\mathrm{F}}=1.205 \theta-46.836\right)$ was faster than that of the hind foot $\left(I_{\mathrm{L}}^{\mathrm{H}}=0.878 \theta-40.634\right)$. On inclinations from $0^{\circ}$ to $30^{\circ}$, the lateral impulses on the front and hind feet pointed to the midline 

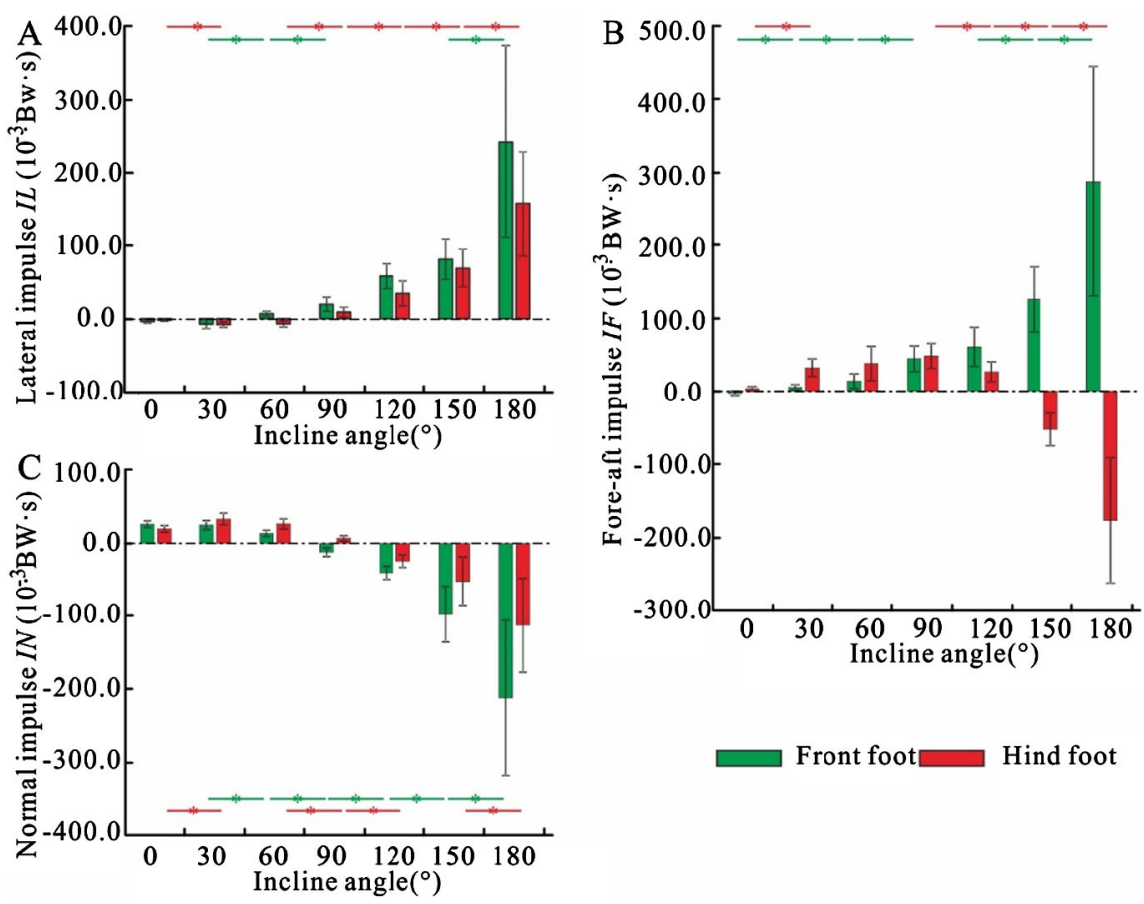

Front foot $\square$ Hind foot

Figure 4. Statistical mean values of the force impulses $\left(I_{\mathrm{L}}, I_{\mathrm{F}}, I_{\mathrm{N}}\right)$ in three directions of the front and hind supporting feet of geckos on different inclines. (A) Lateral force impulse. In order to uniformly describe the lateral force impulses of the left and right legs, the lateral impulse definition of the midline of the body is defined. Negative, otherwise, defined as positive. (B) Fore-aft force impulses. The fore-aft force impulses are the same as the direction of motion, pushing the body forward. (C) Normal force impulse. The negative normal impulse is the adhesion impulse. An asterisk indicates that the corresponding parameters have significant differences between different inclines.

of the body and were similar. On the $60^{\circ}$ inclination, the lateral impulse on the front foot pointed to the outside of the body and the lateral impulse on the hind foot pointed to the midline of the body. When the inclination was greater than $60^{\circ}$, the lateral impulses on the front and hind feet were directed to the outside of the body, and showed a significant difference (Figure 4A).

In the stable movement of the gecko on the inclined surface, the magnitude of the fore-aft impulses of the front foot was increased with the increase of the incline angle $\left(I_{\mathrm{F}}^{\mathrm{F}}=1.442 \theta-48.701\right)$. When moving in the horizontal surface, the front foot produced a negative impulse, the hind foot produced a positive impulse, and its value was greater than the front foot, indicating that the front foot mainly played a braking role when moving on the horizontal 
surface. When the incline angle changed from 30 to $120^{\circ}$, both the front and hind feet produced positive fore-aft impulses which was increased with the increase of the inclination angle, and jointly pushed the body forward; the negative forward-and-backward impulses were generated on the hind legs of the inclined surface of $150^{\circ} \sim 180^{\circ}$ to brake and maintain the stability of the locomotion, and the front foot produced more fore-aft impulses to maintain the locomotion (Figure 4B).

The normal impulses of the front and hind feet were decreased with the increase of the incline angle, and the normal impulse $\left(I_{\mathrm{N}}^{\mathrm{F}}=-1.254 \theta+\right.$ 66.943) of the front foot was decreased faster than the normal impulse of the hind foot $\left(I_{\mathrm{N}}^{\mathrm{H}}=-0.787 \theta+54.588\right)$. On the vertical surface (incline angle $=$ $90^{\circ}$ ), the front foot began to produce normal impulses, exhibiting significant adhesion to pull the body toward the substrate. However, when the incline angle was greater than $120^{\circ}$, the hind foot showed a significant adhesion and produced a negative normal impulse (Figure 4C) (Wang et al., 2015).

\subsection{Stable equilibrium of impulse moment}

During a gait period on a sloping surface, the lateral distance $\vec{a}$ and foreand-aft distance $\vec{b}$ between a set of diagonal stand phases were constant; the direction and size of the fore-and-aft distance were unchanged, and the normal distance between the COM and the moving surface varied slightly with the forward movement of the body. Therefore, in the process of calculating the momentum moment, $\vec{a}, \vec{b}$ and $\vec{h}$ can be used as fixed values. However, the force acting on the foot was a function of time; the lateral and fore-andaft distance between the COM and the hind foot changed as the body moved forward. The direction and size of vector $\vec{a}$ and $\vec{b}$ were unchanged during the duration of a stance phase $\left(T_{\mathrm{S}}\right)$ and were easily obtained from video recording by high definition camera. However, the gecko bent laterally the trunk from one side to the other side to move forward, resulting in the distance from the hind supporting foot to the COM in lateral and fore-aft direction $(\vec{c}$ and $\vec{d}$ ) being both functions of time $(t)$. Meanwhile, the substrate reaction forces acting on foot also changed continually with time. Thus, the calculation of equations (2)-(5) was very difficult. In order to simplify it, variables $\vec{c}$ and $\vec{d}$ were considered constant with time, and the medium value $\left(c_{\text {med }}\right.$ and $d_{\text {med }}$ ) of $\vec{c}$ and $\vec{d}$ were chosen to substitute the time-dependent variable during the stance phase. Experimental results showed that the $c_{m e d}$ was about 
$0.5|\vec{a}|$, the $d_{\text {med }}$ was about $0.3|\vec{b}|$, Thus, equations (2)-(5) could be simplified to equations $(6) \sim(9)$.

$$
\begin{aligned}
& +L_{\mathrm{O}}=\left\{\begin{array}{l}
\left|\int_{0}^{T_{\mathrm{S}}} \vec{h} \times \vec{F}_{\mathrm{F}}^{\mathrm{F}} \mathrm{dt}+\int_{0}^{T_{\mathrm{S}}} \vec{h} \times \vec{F}_{\mathrm{F}}^{\mathrm{H}} \mathrm{dt}+\int_{0}^{T_{\mathrm{S}}} 0.7 \vec{b} \times \vec{F}_{\mathrm{N}}^{\mathrm{F}} \mathrm{dt}\right| \\
\left|\int_{0}^{T_{\mathrm{S}}} \vec{h} \times \vec{F}_{\mathrm{F}}^{\mathrm{F}} \mathrm{dt}+\int_{0}^{T_{\mathrm{S}}} 0.3 \vec{b} \times \vec{F}_{\mathrm{N}}^{\mathrm{H}} \mathrm{dt}\right|
\end{array}\right. \\
& =\left\{\begin{array}{l}
\left|\vec{h} \times \int_{0}^{T_{\mathrm{S}}} \vec{F}_{\mathrm{F}}^{\mathrm{F}} \mathrm{dt}+\vec{h} \times \int_{0}^{T_{\mathrm{S}}} \vec{F}_{\mathrm{F}}^{\mathrm{H}} \mathrm{dt}+0.7 \vec{b} \times \int_{0}^{T_{\mathrm{S}}} \vec{F}_{\mathrm{N}}^{\mathrm{F}} \mathrm{dt}\right| \\
\left|\vec{h} \times \int_{0}^{T_{\mathrm{S}}} \vec{F}_{\mathrm{F}}^{\mathrm{F}} \mathrm{dt}+0.3 \vec{b} \times \int_{0}^{T_{\mathrm{S}}} \vec{F}_{\mathrm{N}}^{\mathrm{H}} \mathrm{dt}\right|
\end{array}\right. \\
& = \begin{cases}\left|\vec{h} \times \vec{I}_{\mathrm{F}}^{\mathrm{F}}+\vec{h} \times \vec{I}_{\mathrm{F}}^{\mathrm{H}}+0.7 \vec{b} \times \vec{I}_{\mathrm{N}}^{\mathrm{F}}\right| & \theta \in\left[0^{\circ}, 60^{\circ}\right] \\
\left|\vec{h} \times \vec{I}_{\mathrm{F}}^{\mathrm{F}}+0.3 \vec{b} \times \vec{I}_{\mathrm{N}}^{\mathrm{H}}\right| & \theta \in\left[90^{\circ}, 180^{\circ}\right]\end{cases} \\
& -L_{\mathrm{O}}= \begin{cases}\left|0.3 \vec{b} \times \vec{I}_{\mathrm{N}}^{\mathrm{H}}\right| & \theta \in\left[0^{\circ}, 60^{\circ}\right] \\
\left|\vec{h} \times \vec{I}_{\mathrm{F}}^{\mathrm{F}}+0.7 \vec{b} \times \vec{I}_{\mathrm{N}}^{\mathrm{F}}\right| & \theta \in\left[90^{\circ}, 180^{\circ}\right]\end{cases} \\
& +L_{\mathrm{R}}= \begin{cases}\left|-0.5 \vec{a} \times \vec{I}_{\mathrm{N}}^{\mathrm{H}}+\vec{h} \times \vec{I}_{\mathrm{L}}^{\mathrm{H}}\right| & \theta \in\left[0^{\circ}, 60^{\circ}\right] \\
\left|-0.5 \vec{a} \times \vec{I}_{\mathrm{N}}^{\mathrm{H}}+\vec{h} \times \vec{I}_{\mathrm{L}}^{\mathrm{F}}\right| & \theta \in\left[90^{\circ}, 180^{\circ}\right]\end{cases} \\
& -L_{\mathrm{R}}= \begin{cases}\left|0.5 \vec{a} \times \vec{I}_{\mathrm{N}}^{\mathrm{F}}+\vec{h} \times \vec{I}_{\mathrm{L}}^{\mathrm{F}}\right| & \theta \in\left[0^{\circ}, 60^{\circ}\right] \\
\left|0.5 \vec{a} \times \vec{I}_{\mathrm{N}}^{\mathrm{F}}+\vec{h} \times \vec{I}_{\mathrm{L}}^{\mathrm{H}}\right| & \theta \in\left[90^{\circ}, 180^{\circ}\right]\end{cases}
\end{aligned}
$$

In equations (6)-(9), $I_{\mathrm{L}}^{\mathrm{H}}, I_{\mathrm{F}}^{\mathrm{H}}$ and $I_{\mathrm{N}}^{\mathrm{H}}$ are the motion reaction impulses acting on the hind foot, respectively. Similarly, $I_{\mathrm{L}}^{\mathrm{F}}, I_{\mathrm{F}}^{\mathrm{F}}$ and $I_{\mathrm{N}}^{\mathrm{F}}$ are, respectively, the motion reaction impulses acting on the front foot.

The overturning impulsive moment and rolling impulsive moment of the gecko moving on different inclined surfaces were related to the actual position of the sole and the COM in the actual movement of the gecko. Experimental results showed that as the gecko moved on different inclined surfaces, the overturning impulse moment was increased with the increase of the inclination angle. When the gecko moved in the horizontal plane, the overturning impulsive moment was the smallest, and there was no difference between the positive and negative impulse moments, which meant that the possibility of overturning was the lowest. When the horizontal plane changed to an incline, the overturning impulse moment significantly increased. There was no significant difference in the overturning impulse moment between the surface of $30^{\circ}$ and the surface of $90^{\circ}(p>0.05)$. There was no significant difference between the positive and negative moments on each incline. On surfaces whose inclination was larger than $90^{\circ}$, the positive and negative overturning moments were increased significantly, and the overturning impulse moment at 
A

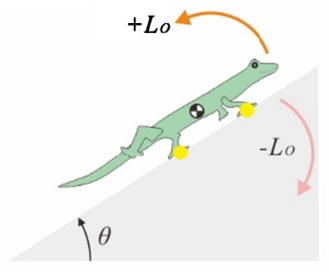

B

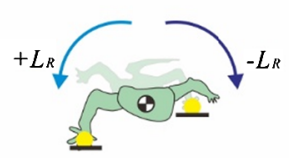

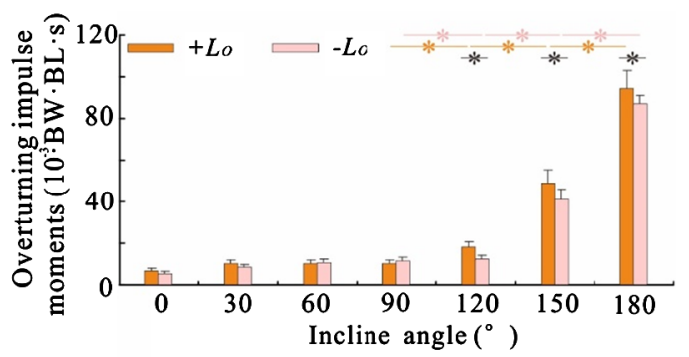

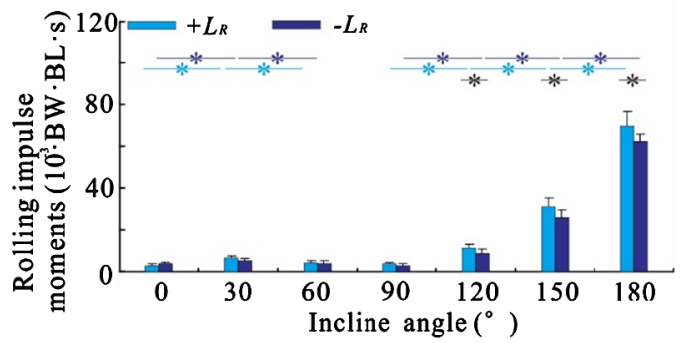

Figure 5. The overturning impulse moment and the rolling impulse moment of geckos around the COM on the different inclines. (A) Overturning impulse moment; an orange-red asterisk indicates that there is a difference in the positive overturning impulse moment between adjacent inclined surfaces, a pink asterisk indicates that there is a difference in the negative overturning impulse moment between adjacent incline surfaces, and a black asterisk indicates that there is a difference between the positive overturning impulse moment and negative overturning impulse moment on inclined surfaces; (B) Rolling impulse moment: a sky-blue asterisk indicates that there is a difference in the positive rolling impulse moment between the adjacent inclined surfaces, a blue asterisk indicates that there is a difference in the negative rolling moment between the adjacent inclined surface, and a black asterisk indicates that there is a difference between the positive rolling impulse moment and the negative rolling impulse moment on inclined surfaces.

$180^{\circ}$ reached 10 times that of the $90^{\circ}$ surface; the positive and negative overturning impulse moments showed significant differences $\left(120^{\circ}: p=0.036\right.$; $\left.150^{\circ}: p=0.022 ; 180^{\circ}: p=0.008\right)$, indicating that the probability of overturning of the surface gecko movement at $120^{\circ}$ surface $-180^{\circ}$ was increasing (Figure 5A).

The rolling impulsive moment was increased first, then decreased and then again increased; when the inclination was larger than $120^{\circ}$, it was increased significantly, and the maximum value of the surface at $180^{\circ}$ reached 15 times of the minimum value of the surface at $90^{\circ}$. On the $0-90^{\circ}$ inclined surface, the positive rolling impulse moment was approximately equal to the rolling impulse moment, while the difference between the positive and negative rolling moments of the inclined surface at $120-180^{\circ}$ was large $\left(120^{\circ}\right.$ surface: 
$\left.p=0.013 ; 150^{\circ}: p=0.037 ; 180^{\circ}: p=0.024\right)$, indicating that the animal would roll from $120-180^{\circ}$ in the two-leg supporting mode (Figure 5B).

\section{Discussion}

Animals balance unstable moments in motion in a variety of ways to achieve motion stability. The titmouse uses the tail to balance the unstable moment caused by the deviation of the centre of mass (COM) from the supporting branches (Norberg, 1986). The climbing animals stretch the limbs as far as possible on the steep inclines, which not only increases the distance of the supporting feet, but also reduces the distance between the center of mass and the base, thereby reducing the possibility of instability (overturning and rolling) on the inclines(Autumn et al., 2006; Han et al., 2011; Endlein et al., 2013). By increasing the distance of the supporting limbs and adopting a crawling posture, small mammals avoid overturning and rolling when moving on the inclines (Higham \& Jayne, 2004; Lammers et al., 2006; Schmidt $\&$ Fischer, 2011). In each gait period, although the maximum rolling impulse moment of the cockroach can cause its body to rotate $360^{\circ}$ around the fore-aft axis, there is no rolling in actual motion. Because two sets of three-legged supporting gait switch rapidly, and the reverse rolling impulse moment generated by the latter three-legged supporting phase balances the rolling impulse moment generated by the former three-legged supporting phase (Ting et al., 1994).

The impulse moment of the gecko moving on different inclined surfaces are related to the actual action position of the feet and the COM in the movement (equations (6)-(9)). The gecko constantly adjusts the distance between the supporting feet to adapt to the change of the incline angle. As the incline angle increases, the diagonal distance between the front and the hind feet increases, and the gecko continues to extend its limbs to the outside to make the body more and more flat. The gecko's low COM and flattened motion pose increase stability in all directions, reducing overturning and rolling caused by its own weight and external disturbances. However, in actual locomotion, the gecko climbs in a crawling posture rather than in an erect posture, and there is a large angle between its foot palm and the sagittal plane of the body (the left and right symmetry planes), so that the COM and the motion plane must keep a certain distance to maintain its crawling posture and the variation range of $h$ is limited; secondly, due to the influence of the gecko's own 
geometry and the normal distance $h$, the lateral and fore-aft distances of the feet increase slightly as the incline angle increases, but the amplitude is also limited (Figure 4C). The maintenance of the dynamic locomotion stability requires not only the adjustment in movement posture but also the coordination of the inter-foot motion function (weight balance mode, driving mode; Wang et al., 2015). When the gecko moves on a $0^{\circ}$ incline, the way of the front foot braking and the hind foot driving will effectively avoid overturning (Herr et al., 2002; Chen et al., 2006); on a shallow incline, the gecko's front foot produces a positive normal impulse to balance the weight and provide positive driving impulses to propel the body. However, excessive normal impulses and driving impulses will produce larger overturning impulse and rolling impulse moments, causing the overturning and rolling (Wang et al., 2015). Thus, similar to other quadrupeds (Dutto et al., 2004; Lammers et al., 2006), the gecko reduces the amount of weight shared by the front limbs, and the weight is mainly borne by the hind limbs, lowering the overturning moment in locomotion, thereby reducing the possibility of overturning; however, when climbing on steeper inclines, the gecko's front foot creates an adhesive force that pulls the body toward the substrate and increase the force arm by extending the body to balance the overturning moment due to gravity (Figure 5A) and reduce the reactionary impact of the foot in motion. The front foot plays an important role in avoiding the animal from overturning. Chameleons and possums have similar characteristics in climbing locomotion (Lammers \& Gauntner, 2008; Krause \& Fischer, 2013).

As the incline angle of the surface increases, the positive and negative overturning impulse moments in each gait period of the gecko are increased, and their difference becomes more prominent. Similarly, the positive and negative rolling moments have the same variation trend. This means that the probability of overturning and rolling in gecko's locomotion is increasing (Figure 5). However, geckos did not obviously overturn or roll in their locomotion during the experiment, because of: (1) adjustment in gait. On steep inclines, the gecko lowers its pace and increases the duty factor. The gecko's gait also shifts from diagonal gait to insect-like triangular gait, i.e. more steps are in the supporting phase at the same time, making the body in a state of multiple support (Autumn et al., 2006; Wang et al., 2011), thereby avoiding overturning and rolling on inclined surfaces; (2) flexibility of body and limbs. The elastic deformation of the limbs and body muscles buffers the 
force and delays the rolling and overturning response(Chen et al., 2006; Bennett et al., 2001), thus achieving stable locomotion. For example, lizards and scorpion muscles can alleviate the impact of force and decrease the effects of unstable impulse moments in motion (Bennett et al., 2001). Although the active swing of the tail has always been important to maintain the stability of the locomotion (Jusufi et al., 2008), experimental observations have shown that the gecko does not swing its tail significantly when moving on the inverted slopes, and since the speed of the gecko on the incline is very low (Figure 5A), the inertial force may not be obvious for maintaining stability.

\section{Conclusions}

This study combines the locomotor behaviour of geckos with mechanics, revealing how geckos control the force and behaviour to avoid overturning and rolling in locomotion. On a shallow incline, the gecko's low COM and flat locomotor posture can effectively improve the stability of the movement. Meanwhile, through the timely conversion of the limb function, the overturning/rolling impulse moments are low, which considerably reduces the probability of overturning/rolling in locomotion. However, on the inverted incline, the difference between the positive and negative overturning moments and the difference between the positive and negative rolling moments are prominent. This means that as the inclined angle increases, especially on the inverted slope, the probability of overturning and rolling in the moving gecko is increasing. Therefore, the gecko makes full use of the flexibility of its body and limbs to delay the occurrence of rolling and overturning, and actively cooperates with the adjustment of the gait, using the alternating change of gait to achieve the avoid of overturning and rolling.

In addition, the moderate abduction of the gecko body can avoid the overturning and rolling of the movement. The extent of the body's abduction is crucial for the animal's locomotion on steep inclines, which indirectly indicates the necessity of the crawling posture to move on steep inclines because the posture can greatly abduct the body and also explains why superb adhesion performance is always embedded in the crawling posture animals.

\section{Acknowledgements}

The first two authors contributed equally to the work. Parts of the data were presented in Wang et al. (2014). Q.X. and Z.W. designed the research; 
Q.X. and Z.W. performed the research; Z.W., W.Z. and J.G. analyzed data; Q.X. wrote the paper. We thank Liuwei Wang and Qijun Jiang for assistance with data collection and manuscript writing. Yi Song and Lei Cai provided insightful comments on the manuscript. This work was supported by the National Natural Science Foundation of China (Grant No. 51975283 and No. 31601870), Natural Science Foundation of Jiangsu Province, China (Grant No. SBK20160800) and the Priority Academic Program Development of Jiangsu Higher Education Institutions (Su Caijiao (2018) No. 192).

\section{References}

Autumn, K., Hsieh, S.T., Dudek, D.M., Chen, J., Chitaphan, C. \& Full, R.J. (2006). Dynamics of geckos running vertically. - J. Exp. Biol. 209: 260-272.

Bennett, W.O., Simons, R.S. \& Brainerd, E.L. (2001). Twisting and bending: the functional role of salamander lateral hypaxial musculature during locomotion. - J. Exp. Biol. 204: 1979-1989.

Bi, J., Wang, Y., Li, S. \& Zeng, Z. (2015). Is habitat preference associated with locomotor performance in multiocellated racerunners (Eremias multiocellata) from a desert steppe. — Asian Herpetol. Res. 6: 143-149.

Chen, J.J., Peattie, A.M., Autumn, K. \& Full, R.J. (2006). Differential leg function in a sprawled-posture quadrupedal trotter. — J. Exp. Biol. 209: 249-259.

Dai, Z., Wang, Z. \& Ji, A. (2011). Dynamics of gecko locomotion: a force-measuring array to measure 3D reaction forces. - J. Exp. Biol. 214: 703-708.

Dickinson, M.H., Farley, C.T., Full, R.J., Koehl, M.A.R., Kram, R. \& Lehman, S. (2000). How animals move: an integrative view. - Science 288: 100-106.

Dutto, D.J., Hoyt, D.F., Cogger, E.A. \& Wickler, S.J. (2004). Ground reaction forces in horses trotting up an incline and on the level over a range of speeds. - J. Exp. Biol. 207: 35073514.

Endlein, T., Ji, A., Samuel, D., Yao, N., Wang, Z., Barnes, W.J.P. \& Dai, Z. (2013). Sticking like sticky tape: tree frogs use friction forces to enhance attachment on overhanging surfaces. - J. R. Soc. Interface 10: 20120838.

Goldman, D.I., Chen, T.S., Dudek, D.M. \& Full, R.J. (2006). Dynamics of rapid vertical climbing in cockroaches reveals a template. - J. Exp. Biol. 209: 2990-3000.

Han, L., Wang, Z., Ji, A. \& Dai, Z. (2011). Grip and detachment of locusts on inverted sandpaper substrates. - Bioinspir. Biomim. 6: 046005.

Herr, H.M., Huang, G.T. \& McMahon, T.A. (2002). A model of scale effects in mammalian quadrupedal running. - J. Exp. Biol. 205: 959-967.

Higham, T.E. \& Jayne, B.C. (2004). Locomotion of lizards on inclines and perches: hindlimb kinematics of an arboreal specialist and a terrestrial generalist. - J. Exp. Biol. 207: 233248.

Holmes, P., Full, R.J., Koditschek, D. \& Guckenheimer, J. (2006). The dynamics of legged locomotion: models, analyses, and challenges. — SIAM Rev. 48: 207-304. 
Jusufi, A., Goldman, D.I., Revzen, S. \& Full, R.J. (2008). Active tails enhance arboreal acrobatics in geckos. — Proc. Natl. Acad. Sci. USA 105: 4215-4219.

Krause, C. \& Fischer, M.S. (2013). Biodynamics of climbing: effects of substrate orientation on the locomotion of a highly arboreal lizard (Chamaeleo calyptratus). — J. Exp. Biol. 216: $1448-1457$.

Lammers, A.R. \& Gauntner, T. (2008). Mechanics of torque generation during quadrupedal arboreal locomotion. - J. Biomech. 41: 2388-2395.

Lammers, A.R., Earls, K.D. \& Biknevicius, A.R. (2006). Locomotor kinetics and kinematics on inclines and declines in the gray short-tailed opossum Monodelphis domestica. J. Exp. Biol. 209: 4154-4166.

Lee, D.V. (2011). Effects of grade and mass distribution on the mechanics of trotting in dogs. — J. Exp. Biol. 214: 402-411.

Lee, D.V., Bertram, J.E. \& Todhunter, R.J. (1999). Acceleration and balance in trotting dogs. - J. Exp. Biol. 202: 3565-3573.

Martinez, M.M., Full, R.J. \& Koehl, M.A. (1998). Underwater punting by an intertidal crab: a novel gait revealed by the kinematics of pedestrian locomotion in air versus water. J. Exp. Biol. 201: 2609-2623.

Norberg, R.Å. (1986). Treecreeper climbing; mechanics, energetics, and structural adaptations. - Ornis Scand. 17: 191-209.

Schmidt, A. \& Fischer, M.S. (2011). The kinematic consequences of locomotion on sloped arboreal substrates in a generalized (Rattus norvegicus) and a specialized (Sciurus vulgaris) rodent. — J. Exp. Biol. 214: 2544-2559.

Taylor, J.N., Ternes, W.M. \& Lattanzio, M.S. (2018). Natural selection favors local specialization in a widespread habitat generalist. - Evolution 72: 2090-2099.

Ting, L.H., Blickhan, R. \& Full, R.J. (1994). Dynamic and static stability in hexapedal runners. - J. Exp. Biol. 197: 251-269.

Wakeling, J.M. \& Johnston, I.A. (1999). Body bending during fast-starts in fish can be explained in terms of muscle torque and hydrodynamic resistance. - J. Exp. Biol. 202: 675-682.

Wang, Z., Wang, J., Ji, A., Zhang, Y. \& Dai, Z. (2011). Behavior and dynamics of gecko's locomotion: the effects of moving directions on a vertical surface. - Chin. Sci. Bull. 56: 573-583.

Wang, Z.Y., Ji, A.H., Endlein, T., Li, W., Samuel, D. \& Dai, Z.D. (2014). Locomotor kinematics of the gecko (Tokay gecko) upon challenge with various inclines. - Chin. Sci. Bull. 59: 4568-4577.

Wang, Z., Dai, Z., Li, W., Ji, A. \& Wang, W. (2015). How do the substrate reaction forces acting on a gecko's limbs respond to inclines? — Sci. Nat. 102: 7. 